\title{
Una inscripción mágica de Panticapeo única y los enemigos desconocidos*
}

\author{
Eleni Chronopoulou \\ Università degli Studi di Firenze \\ eleni.chronopoulou@unifi.it \\ ORCID: https://orcid.org/0000-0003-1730-3334
}

\section{A unique magical inscription from Panticapaeum and the unknown enemies}

\begin{abstract}
En este artículo se estudia una inscripción mágica de la antigua necrópolis de Panticapeo que contiene inscrita diecisiete veces la palabra óvóvvuos junto a unas letras sin sentido aparente. Se hacen unas observaciones sobre la lectura, unas anotaciones sobre las secuencias de las letras sin sentido y una nueva propuesta sobre su finalidad.
\end{abstract}

Palabras Clave: inscripción; mágica; Panticapeo; anónimos.
The present article studies a magical inscription from the ancient necropolis of Panticapaeum that

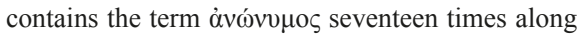
with some letters without obvious meaning, making observations on the reading of the text and annotations about the letters without meaning and offering a new proposal concerning its purpose.

Key words: inscription; magical; Panticapaeum; anonymous.

Cómo citar este artículo / Citation: Chronopoulou, Eleni 2019: «Una inscripción mágica de Panticapeo única y los enemigos desconocidos», Emerita 87 (1), pp. 73-82.

En el año 2011, en Panticapeo (actual Kerch en Crimea), en la cima del Monte Mitrídates, donde se encuentra la antigua necrópolis, se halló una inscripción que fue editada por A. Belousov y N. Fedoseev en el año $2014^{1}$.

* Este artículo ha sido elaborado en el marco de los proyectos de investigación FFI201787558-P (AEI/FEDER, UE) financiado por el MINECO, y «La magia aggressiva nel mondo antico: lessico e formulario dei testi in greco» de la Universidad de Florencia. Más datos en https://www.progettomagia.unifi.it/vp-17-obiettivi.html. La autora agradece a los evaluadores anónimos y al editor de Emerita sus sugerencias y su ayuda en la revisión del español.

${ }^{1}$ Belousov y Fedoseev 2014, pp. 145-148. 
Como señalan los editores, se trata de una inscripción única y misteriosa a la vez. Tiene $16 \mathrm{~cm}$ de longitud y su anchura oscila entre 2.5 y $6.5 \mathrm{~cm}$. Está plegada cinco veces y el agujero que hay en su superficie indica que fue perforada con un clavo. En esta lámina de plomo aparece inscrita múltiples

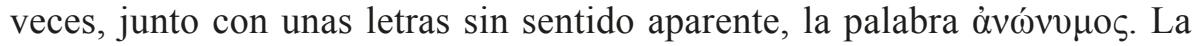
paleografía de la inscripción no revela mucho sobre la época de su producción y los editores proponen el siglo III a. C. ${ }^{2}$ como fecha post quem. El hecho de que sea de plomo, que esté plegada y que haya sido perforada, además de haberse depositado en un cementerio, son fuertes indicios de que se trata de una inscripción mágica, y concretamente de una defixio. Sin embargo, aunque el formato es típico de una defixio, el contenido resulta

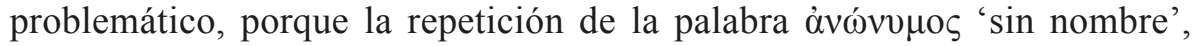
'anónimo' no tiene paralelo entre los centenares de defixiones descubiertas hasta el momento.

Por lo que se refiere al texto de la tablilla, la transcripción que presento aquí está basada en el facsímil que aparece en la primera edición ${ }^{3}$. Las diferencias de lectura se discuten más adelante.

Col. 1

1. åvóvvนo

2. $\dot{\alpha} v \omega ́ v v \mu \mathrm{o}[\zeta]$

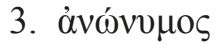

4. $\dot{\alpha}[v] \omega ́ v v \mu о \varsigma$ OP

5. $\dot{\alpha} v \omega ́ v v \mu[o \varsigma]$

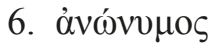

\section{Col. 2}

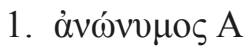

2. $\dot{\alpha} v \omega ́ v 0 \mu \circ \Delta \mathrm{A}$

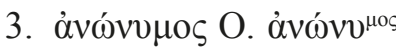

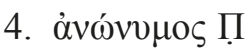

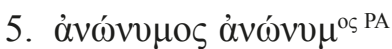

6. $\dot{\alpha} v \omega \dot{v} v \mu \mu \varsigma \Sigma$

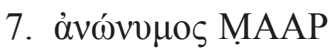

8. $\dot{\alpha} v \omega ́ v v \mu \circ \mathrm{T}$ !

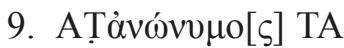

Una primera observación es que la segunda columna está mucho más comprimida y las líneas no tienen correspondencia exacta. En concreto:

\footnotetext{
2 Belousov y Fedoseev 2014, p. 145.

${ }^{3}$ Para una mejor comprensión de la tablilla y de las diferencias invito al lector a que consulte dicho facsímil.
} 
a) La sílaba OP que los editores ponen al principio de la 1.8 de la col. 2

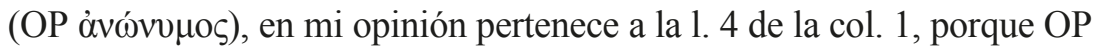
está antes de la línea recta que forman las letras iniciales de la col. 2.

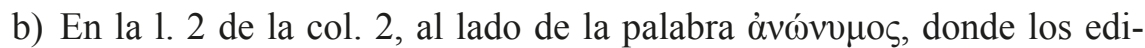
tores ponen una interrogación, se ve claramente una A y una letra que contiene una parte de forma triangular, que puede ser una $\Delta$, una $\mathrm{M}$ o incluso una $\mathrm{N}$.

c) En la 1. 3 de la col. 2, la letra con el punto debajo tras la $\mathrm{O}$ tiene la forma de una $\Lambda$, con la segunda línea más corta. Los editores la interpretan como una P. Sin llegar a rechazar totalmente esta propuesta, me parece poco probable, porque las otras $\mathrm{P}$ que aparecen en la tablilla están escritas con el semicírculo muy redondo. En esa misma línea, la

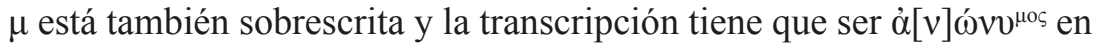
lugar de $\dot{\alpha}[v] \omega ́ v v \mu^{\circ \varsigma}$, como proponen los editores.

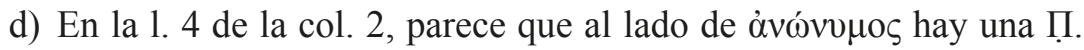

e) Una diferencia fundamental se da en las 1. 4-5 de la col. 2. Los editores

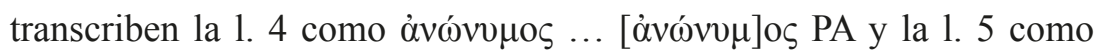
$\dot{\alpha} v \omega ́ v v \mu$ o $\ldots \dot{\alpha} v \omega ́ v v \mu_{[\circ]]}$. No creo que haya espacio en la 1.4 para que quepan seis letras. En el mismo espacio de arriba caben tres, quizá cuatro letras. Mi opinión es que el escriba no tenía suficiente espacio en la 1.5 para terminar la palabra y utilizó el espacio libre arriba, como hizo también en la 1. 3, donde la sílaba suprascripta ${ }^{\mu \circ \varsigma}$ se escribe entre

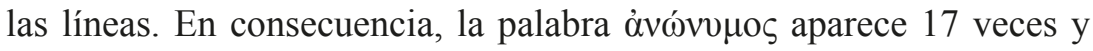
no 18 , como sugerían los editores.

f) En las 11.8 y 9, donde leo T, los primeros editores interpretaban que hay una cruz. La presencia de una cruz en este contexto es muy poco probable. En otras laminillas (véanse los facsímiles en Wünsch) se puede apreciar que la $\chi$ algunas veces tiene la forma de una cruz ${ }^{4} \mathrm{o}$ de una $\mathrm{T}$, como se ve en una defixio de Sicilia ${ }^{5}$.

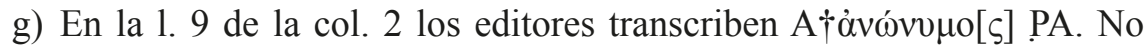
excluyo la posibilidad de que AT pertenezca al final de la 1.5 de la col. 1. La P, tal y como aparece en el facsímil de la inscripción, me parece más una T.

${ }^{4}$ Véase DTA $65 ; 66 ; 77 ; 88 ; 90$.

5 Jordan-Rocca-Threatte 2014, pp. 231-236. 
Hay que destacar en esta inscripción las letras sin sentido aparente que acompañan a veces a la palabra ỏvóvvuos. Parece que no están escritas aleatoriamente allí donde había un espacio vacío, porque entre la primera columna y la segunda hay bastante espacio, pero aparece sólo la sílaba OP en la 1. 4 de la col. 1. Desgraciadamente, como admiten los editores, no nos resulta fácil entender su función. Es probable que sean abreviaturas o voces mágicas o, mejor, letras mágicas. El paralelo más cercano y seguro que he podido encontrar (porque su lectura no presenta demasiados problemas) es el de DTA 11 , que contiene una lista de nombres de víctimas. Al lado de los nombres, en las 11.2 y 3 , aparecen las letras $\Delta \mathrm{N}$ y $\mathrm{MM}$ respectivamente. Wünsch los comentó del siguiente modo: $« \Delta \mathrm{N}$ MM litterae additae uidentur, ut magicam augerent speciem». Entre estas letras sin sentido que pueden tener valor mágico encontramos MAAP en una secuencia de uoces magicae en DT 38 y también en PGM XII $168^{6}$.

La perplejidad de los editores ante una pieza tan única está absolutamente justificada. Para dar una explicación de este interesante hallazgo, proponen, no sin ciertas dudas, que la palabra ỏvóvvuos debe referirse a entidades intermedias y que la inscripción es una invocación a un daimon o a un nekydai$m^{7}{ }^{7}$. Su conclusión se basa en dos argumentos principales: a) en la Antigüedad había divinidades cuyo culto se hacía bajo anonimato, así como seres intermedios o espíritus que se invocaban con nombres genéricos como ö́por

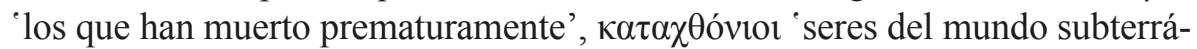

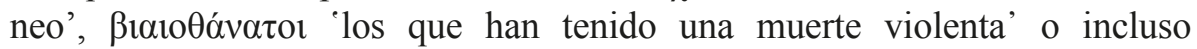

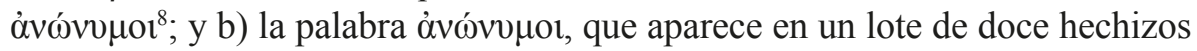

${ }^{6}$ La secuencia de las letras $\mu \alpha \rho$ es muy frecuente en las uoces magicae, pero $\mu \alpha \alpha \rho$ lo he encontrado solamente en estos dos textos. OP aparece frecuentemente al final de palabras

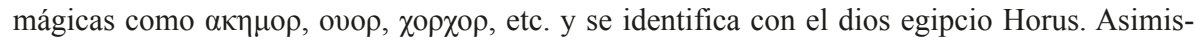
mo, PA se encuentra en los papiros mágicos y se identifica con el dios egipcio Ra. El lector puede consultar también estas entradas en el III volumen de índices de PGM: Preisendanz 1941, pp. 265 y 229. Sin embargo, si estas identificaciones son correctas, parece que fuera del contexto egipcio no tienen sentido.

${ }^{7}$ En un artículo posterior Belousov 2016, p. 42 ha admitido con respecto a esta inscripción: «I quite arbitrarily put under the heading inuocatio ad inferos».

${ }^{8}$ Ogden 2002, p. 146 habla de cuatro categorías de espíritus ('ghosts') que eran convenientes para su uso en la magia: a) los aoroi 'los que han muerto jóvenes' como los bebés o los niños; b) los biaiothanatoi 'los que han muerto de manera violenta', como los muertos en combate; c) los agamoi 'los que han muerto antes de casarse' y d) los ataphoi 'los que 
judiciales parecidos, hallados en Kourion (Chipre), se emplea para referirse a dioses subterráneos ${ }^{9}$. Para reforzar su argumentación mencionan también una tabella iudiciaria de Olbia en la que se invoca a un espíritu desconocido empleando magia analógica. La analogía es que «como nosotros no te conocemos, así Éupolis .... ${ }^{10}$. Basándose en el hecho de que los ejemplos análogos más cercanos resultan ser tabellae iudiciariae, formulan la hipótesis de que podría tratarse también en nuestro caso de una maldición de este tipo.

Sin embargo, hay que admitir que esta hipótesis choca bastante con la estructura establecida de las defixiones.

a) Efectivamente, en la Antigüedad había deidades cuyo nombre no se conocía y cuyo culto se realizaba bajo anonimato porque no hacía falta mencionar el nombre ${ }^{11}$ o por miedo ${ }^{12}$ o incluso porque se desconocía completamente. En los últimos dos casos se empleaban los

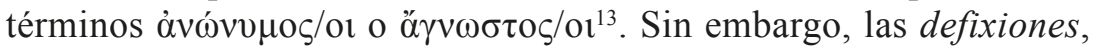
especialmente las más antiguas ${ }^{14}$, correspondientes a la época clásica y helenística, contienen inscritos los nombres de las víctimas, no los nombres de los dioses o de los daimones invocados. No hay paralelo de tablillas mágicas que den cuenta solamente de los nombres de divinidades o de espíritus invocados ${ }^{15}$. Además, la hipótesis

han quedado sin entierro o sin recibir los honores funerarios'. Para los aoroi véase también Johnston 1999, pp. 127-150.

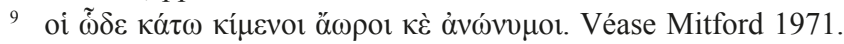

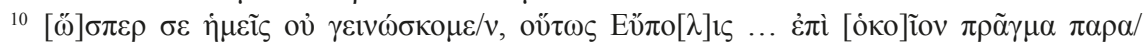

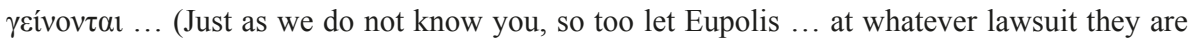
present ...). Tanto la edición como la traducción son de Jordan 1997, pp. 212-219. Sobre esta tablilla véase también Shkorpil 1908, pp. 71-72 y Dubois 1996, pp. 176-178.

${ }^{11}$ A veces los dioses eran tan conocidos por su dedicación en algo que no hacía falta ni mencionar su nombre en una invocación como en el proemio de la Ilíada donde la Musa es simplemente invocada por un $\theta \varepsilon \alpha ́$.

${ }_{12}$ A veces su nombre era tan terrible y provocaba tanto miedo que preferían evitar pronunciarlo, como en el caso de las Erinias, a las que Eurípides llama ỏvóvouor $\theta \varepsilon \alpha i ́$ (IT 944). Véase Johnston 1999, pp. 270-280; Robertson 2010, p. 89 y Athanassakis y Wolkow 2013, p. 190.

${ }_{13}$ Sobre el dios/los dioses desconocido/s son fundamentales los estudios de Norden 1913, pp. 115-124; Van der Horst 1988, pp. 19-42 y Henrichs 1994, pp. 27-58.

14 Véase Gager 1999, p. 5.

${ }^{15}$ Hay que admitir que hay tablillas cuyo contenido sigue siendo dudoso. Las secuencias de las letras que los editores han descifrado no coinciden con una secuencia de letras de la 
de que el defigens utiliza todo el espacio de la tablilla para invocar a diecisiete entidades diferentes o a la misma entidad diecisiete veces sin saber su(s) nombre(s) resulta problemática. En los textos de Kourion se emplea la palabra en plural para agrupar todas las entidades de este tipo ${ }^{16}$, como suele hacerse también con otro tipo de nombres genéricos y colectivos ${ }^{17}$. Es importante tener en cuenta que ninguna de las defixiones que tenemos hasta ahora de esta región ${ }^{18}$ incluye una invocación a daimones u otro tipo de espíritus. Por el contrario, hay numerosos ejemplos que contienen solamente los nombres de las personas contra las que iba dirigida la maldición. Recientemente se ha publicado una defixio procedente de la misma necrópolis con una invocación a Hécate ${ }^{19}$, fechada en el siglo $\mathrm{V}$ a. C. Por el momento es la única en cuyo texto aparece también una invocación a una divinidad.

b) La intención fundamental para la fabricación de una defixio es la de maldecir a alguien y provocarle daño. Si no existe esta intención, no se puede clasificar como tal. Por lo tanto es necesario que haya una víctima. Aunque es cierto que existen tablillas que por su formato, contexto y soporte parecen una defixio, si bien su contenido es incomprensible, la inmensa mayoría de las tablillas de magia aplicada contienen los nombres de las víctimas, del mismo modo que los formularios marcan el espacio donde tiene que pronunciarse el nombre de la víctima con ó $\delta \varepsilon \tilde{v} \alpha / \dot{\eta} \delta \varepsilon \tilde{v} \alpha$, para que el usuario ponga el nombre de la persona a la que quiere maldecir o «atar» ${ }^{20}$. Incluso en la misma tablilla que los editores dan como el ejemplo análogo más próximo, las

\footnotetext{
lengua griega o no son suficientes para que se logre una lectura. En DTA 113, por ejemplo, se identifican las letras, pero no tienen sentido y Wünsch las consideró «litterae magicae». Véase Gager 1999, p. 6.

${ }^{16}$ Cuando la invocación implica una multitud de estos seres se utiliza la palabra en plural,

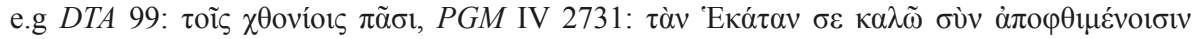

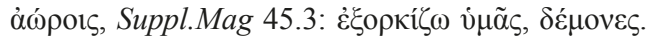

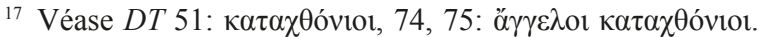

18 Están publicadas más de 40 inscripciones de Olbia y más de 30 de la región del Bósforo.

19 Belousov y Fedoseev 2016, pp. 135-140.

${ }^{20}$ Como subraya Gager 1999, p. 14, no todas las tablillas conservan escritos los nombres. Algunas eran preparadas de antemano y se dejaba un espacio en blanco para añadir después el nombre de la víctima. Si este era largo, se escribía muy comprimido o abreviado.
} 
víctimas son nombradas y claramente citadas. En los casos de magia aplicada se observa que la composición de una defixio exigía la presencia de una fórmula mágica que contenía verbos con sentido de su-

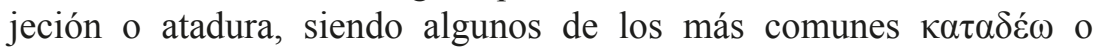

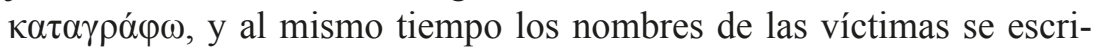
bían en la tablilla, para que quedaran «atados» para siempre o durante el periodo en que la tablilla permaneciese escondida ${ }^{21}$.

c) El término ỏvóvouo vocación, sería de esperar que apareciera en vocativo ${ }^{22} \mathrm{o}$, en el caso de que hubiera un verbo de invocación o conjuro en la fórmula oral (que no aparece en la tablilla), el término tendría que ir en acusativo ${ }^{23}$. En las defixiones los nombres de las víctimas aparecen en nominativo cuando falta el verbo de la maldición ${ }^{24}$.

La estructura establecida de las defixiones apunta hacia hombres y no divinidades o seres intermedios como los aoroi o los biaiothanatoi. Sería más

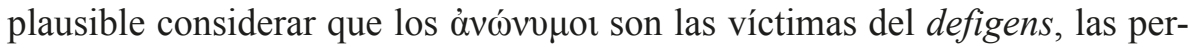
sonas contra las que se dirige la maldición. Sin embargo, esta propuesta plantea también un problema: en la magia el nombre de la víctima tiene la máxima importancia para que sea efectiva la maldición. Con el paso del tiempo se aprecia una mayor preocupación por la precisión en la identidad de la víctima, por lo que estos nombres van acompañados del nombre del padre o, más frecuentemente, de la madre, que, según Chaniotis, da la máxima precisión a la identidad de la víctima, dado que la madre es siempre segura, pero el padre no ${ }^{25}$.

Pero hay que pensar que seguramente habría casos en que el defigens no conociera el nombre o los nombres de sus enemigos. Muy característico es el caso de las llamadas «prayers for justice» o «judicial prayers». En esta categoría de defixiones las «víctimas» que se supone que han provocado mal al defigens son desconocidas y en las maldiciones son mencionadas como

${ }^{21}$ Véase Gager 1999, p. 7.

22 Véase DTA 88; 89; 93; 94. En DTA 101 Hermes es invocado en nominativo, pero no es una defixio que contenga sólo una lista de nombres.

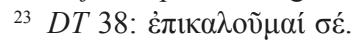

${ }^{24}$ Véase por ejemplo DTA 1-31; DT 60-63.

${ }^{25}$ Vease Chaniotis 2008, p. 58. 
«los que han robado», «ellos», «esta persona» «contra aquellos que» ${ }^{26}$. Son personas que han robado algo al defigens, le han hecho alguna acusación anónima falsa ${ }^{27}$, declaran contra él en un juicio ${ }^{28}$ o quizás el defigens teme que le hayan maldecido primero ellos. Como se puede ver, para todos estos no se menciona el nombre y, cuando el que hace la maldición cree que sus adversarios son muchos, también se emplea el plural en vez de maldecirlos uno por uno. Sin embargo, la estructura de nuestra defixio de Panticapeo parece que corresponde a una etapa de magia menos elaborada en lo que se refiere al texto de la tablilla.

Resumiendo, considero más probable que los anónimos de esta tablilla sean personas adversarias del defigens. El hecho de que la palabra ỏvóvvuos se repita 17 veces puede significar tres cosas: a) que el defigens no conoce cuántos son sus enemigos y escribe todos los que caben en la tablilla;

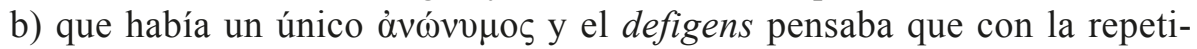
ción se reforzaba la maldición ${ }^{29}$; o c) que sabía exactamente cuántos eran pero no conocía sus nombres. En mi opinión, la primera opción es la más probable.

\section{BiBLIOGRAFÍA}

Athanassakis, A. N. y Wolkow, B. M. 2013: The Orphic Hymns, Baltimore.

Belousov, A. 2016: «Some observations on defixiones from Olbia and Bosporus», en Manoledakis, M. (ed.), Black Sea in the light of New Archaeological data and Therotical Approaches: Proceedings of the $2^{\text {nd }}$ International Workshop on the Black Sea in Antiquity held in Thessaloniki, 18-20 September 2015, Oxford.

\footnotetext{
${ }^{26}$ Una defixio muy interesante a este respecto es la de $S E G$ XXX 326, en la que se pide a Hécate: «ata a los ladrones o al ladrón que robó las cosas mencionadas».

27 Aunque en general las acusaciones anónimas no eran admitidas ni en Grecia ni en Roma, seguramente habría casos. Véase Benko 1980, pp. 9-10; Liebs 2012, pp. 135-136.

${ }^{28}$ E.g. en maldiciones encontradas en Mitilene hay listas de nombres y después frases

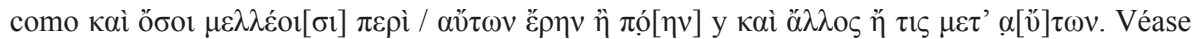
Curbera y Jordan 1998, pp. 31-41. Véase también DT 35 y DTA 66.

${ }^{29}$ Hay maldiciones en las cuales se repite en la misma tablilla el nombre de la víctima o el resultado esperado, quizá con la intención de que sea más efectiva, como por ejemplo en $S G D 22$ donde Filóstrata es maldecida múltiples veces y la maldición de las partes de su cuerpo se repite cuatro veces. Otro ejemplo es DTA 94. También hay maldiciones contra la misma persona en más de una tablilla, como, por ejemplo, DTA 31 i y 31 ii.
} 
Belousov, A. y Fedoseev, N. 2014: «A new magical inscription from Panticapaeum's necropolis», ZPE 190, pp. 145-148.

Belousov, A. y Fedoseev, N. 2016: «A New Defixio From the Ancient Panticapaeum Necropolis», The Journal of Ancient History (VDI) 1, pp. 135-140.

Benko, S. 1980: Pagan Rome and the Early Christians, Indianápolis.

Chaniotis, A. 2008: «Like an unjustified curse: the archaeology of a simile», en Avagianou, A. (ed.), Magic in Ancient Greece, Atenas.

Curbera, J. y Jordan, D. 1998: «Curse Tablets from Mytilene», Phoenix 52, pp. 31-41.

DT: Audollent, A. 1904: Defixionum tabellae quotquot innotuerunt tam in Graecis orientis quam in totius occidentis partibus praeter Atticas in Corpore Inscriptionum Atticarum editas, París.

DTA: Wünsch, R. 1897: Defixionum Tabellae Atticarum, Berlín.

Dubois, L. 1996: Inscriptions grecques dialectales d'Olbia du Pont, Ginebra.

Gager, G. J. 1999: Curse Tablets and Binding Spells from the Ancient World, Oxford.

Henrichs, A. 1994: «Anonymity and Polarity: Unknown Gods and Nameless Altars at the Areopagos», ICS 19, pp. 27-58.

Johnston, S. I. 1999: Restless Dead: Encounters Between the Living and the Dead in Ancient Greece, Berkeley.

Jordan, D. R., 1997: «An Address to a Ghost at Olbia», Mnemosyne 50 (2), pp. 212-219.

Jordan, D. R., Rocca, G. y Threatte, L. 2014: «Una nuova defixio dalla Sicilia (Schøyen Collection MS 1700)», ZPE 188, pp. 231-236.

Liebs, D. 2017: Summoned to the Roman Courts: Famous Trials from Antiquity, Berkeley - Los Angeles - Londres (trad. R. L. R. Garber).

Mitford, T. B. 1971: The Inscriptions of Kourion, Filadelfia.

Norden, E. 1913: Agnostos Theos. Untersuchungen zur Formengeschichte religiöser Rede, Lipsia-Berlín.

Ogden, D. 2002: Magic, Witchcraft, and Ghosts in the Greek and Roman Worlds: A Sourcebook, Oxford.

PGM: Preisendanz, K. 1973-1974: Papyri Graecae Magicae. Die griechischen Zauberpapyri. 2 vol., $2^{\mathrm{a}}$. ed. revisada por A. Henrichs. Stuttgart.

Preisendanz, K. 1941: Papyri Graecae Magicae III: Index (pruebas finales solo).

Robertson, N. 2010: Religion and Reconciliation in Greek Cities. The Sacred Laws of Selinus and Cyrene, Oxford.

SEG: Supplementum Epigraphicum Graecum.

$S G D$ : Jordan, D. R. 1985: «A Survey of Greek Defixiones Not Included in the Special Corpora», GRBS 26, pp. 151-197.

Shkorpil, V. 1908: «Three Lead Tablets with Inscriptions from Olbia», IAK 27, pp. 71-72. 
Suppl.Mag: Daniel, R. W. y Maltomini, F. 1990-1992: Supplementum Magicum. 2 vols., Opladen.

Van der Horst, P. W. 1988: «The Unknown God (Acts 17.23)» en Van den Broek, R. B. (ed.), Knowledge of God in the Greco-Roman World, Leiden.

Fecha de recepción de la primera versión del artículo: 15/01/2019

Fecha de aceptación: 21/03/2019

Fecha de recepción de la versión definitiva: 02/04/2019 